\title{
INFLUENCE OF INFORMATION COLLECTION STRATEGY ON DESIGNER'S MENTAL STRESS
}

\author{
Zhao, Mengting; Zeng, Yong \\ Concordia University, Canada
}

\begin{abstract}
Information collection may affect the design quality and designer's performance through changing the structure of information and the way how information is searched and organized. Based on the theoretical analysis conducted by Wang et al., the present work continues to investigate the influence of designer's natural choice of information collection strategy on his/her mental stress both theoretically and empirically. Designers' stresses are quantified from HRV data and are compared under different information collection strategies.
\end{abstract}

Keywords: Information collection strategy, Mental stress, Bio-inspired design / biomimetics, Design process, Information management

\section{Contact:}

Zhao, Mengting

Concordia University

CIISE

Canada

z_mengti@encs.concordia.ca

Cite this article: Zhao, M., Zeng, Y. (2019) 'Influence of Information Collection Strategy on Designer's Mental Stress', in Proceedings of the 22nd International Conference on Engineering Design (ICED19), Delft, The Netherlands, 5-8 August 2019. DOI:10.1017/dsi.2019.184 


\section{INTRODUCTION}

Information collection strategies are believed to be related to the quality of decisions as one's choice of searching strategies differed from time to time during the entire decision-making process (Cook, 1993). Similar studies have supported this claim by analysing the influence of information collection strategies on consumer's shopping choices, student's performance in school, as well as human performance in other cases (Eysenbach and Köhler, 2002; Scott and O'Sullivan, 2005; Wilczynski and Haynes, 2004). When it comes to design activities, information collection may also affect the design quality and designer's performance through changing the structure of information and the way how information is searched and organized (Bardwell, 1991; Wang et al., 2015). The information identified will subsequently reformulate the design problem (Zeng, 2004). According to Environment-Based Design (Zeng, 2004), searching for information during a design process is to search for environment components and their relationships, where the environment includes existing knowledge about customers, technologies, and other relevant product related components. From this point of view, the application of different information collection strategies corresponds to the order in which different objects are studied. Continuous efforts have been made on investigating the nature of information collection strategies (Belkin et al., 1995; Cook, 1993; Leber and Egeth, 2006). Moreover, researchers have succeeded in recording people's information collection patterns using techniques like eye movements tracing (Broniarczyk and Griffin, 2014; Russo and Dosher, 1983; Vu et al., 2016), whereas the present paper concentrates on how designer's performances are affected while applying different information collection strategies.

Wang et al. conducted a theoretical analysis of how design creativity is influenced by the applied information collection strategy through mental stress (Wang et al., 2015). Different models and change of mental stresses have been derived from analysing each influential factor of mental stress under scenarios where different information collection strategies are applied. In the meanwhile, certain HRV (Heart Rate Variability) features are identified as useful indicators, such as the mean of RR intervals (mRR), and power spectra in very low (VLF), low (LF), and high (HF) frequency ranges, are believed to be affected by mental stress (Hjortskov et al., 2004; Kumar et al., 2007; Salahuddin et al., 2007). The LF/HF ratio derived from the mentioned features, which is sometimes noted as Sympathovagal Balance index (SVI), has already been used as an indicator of mental stress by scholars as it is positively related to mental stress level (Boonnithi and Phongsuphap, 2011; Healey and Picard, 2005; Karthikeyan et al., 2011).

\section{MODEL BASED STRESS ANALYSIS UNDER DIFFERENT INFORMATION COLLECTION STRATEGIES}

Wang et al. (Wang et al., 2015) compared three information collection strategies, namely depth-first, breadth-first and hybrid. As illustrated in Figure 1, information is collected from the top layer to the end along each branch before moving to another branch under the depth-first strategy, whereas under the breadth-first strategy, information at the same level within different branches will be collected before moving to a deeper level. Then the hybrid strategy can be seen as a compromise of both depthfirst and breadth-first strategies.
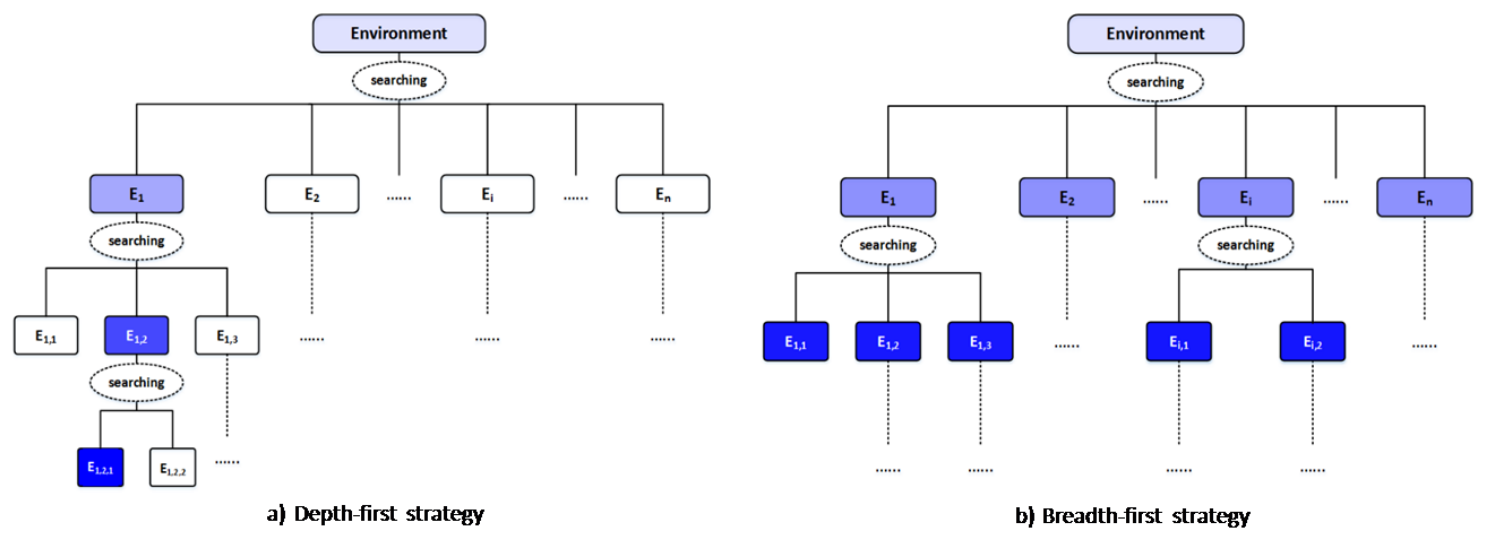

Figure 1. Depth-first and breadth-first information collection strategies 
The analysis conducted by Wang et al. (Wang et al., 2015) is based on the two postulates of the design creativity model proposed in (Nguyen and Zeng, 2012), namely the postulate of nonlinear design dynamics and the postulate of relationship between design activity and designer's mental stress. Wang et al. explained why information search strategies during early stage have a significant impact on designer's performance and design solutions based on the first postulate which implies the sensitive dependence of design on initial conditions. The second postulate indicated that design creativity is related to designer's mental stress through an inverse U shaped curve and stress is influenced by designer's workload and mental capacity (Eq. (1), (Nguyen and Zeng, 2012)). Based on the mentioned two postulates, the influential factors of mental stress, including workload, knowledge, skills, and affect, were analysed under different scenarios corresponding to different information collection strategies in (Wang et al., 2015).

$$
\text { Mental stress }=\frac{\text { Workload }}{(\text { knowledge }+ \text { skills }) * \text { affect }}
$$

In the present paper, we will continue with the analysis conducted by Wang et al. (Wang et al., 2015), which leads to a hypothesis which will be tested with a selected case where data were collected from a design experiment conducted at Concordia University (Nguyen et al., 2013). Each participant was assigned the same design task so that the workload was the same for all participants in the beginning of the design. Besides, participants were given unlimited time to develop their design solutions and they were clearly told that their solutions would not be marked. The objective is to reduce the possibility that participants experience extreme emotions (affect) during the entire process. Participants were using their comfortable approaches during the experiment as we did not specify the strategy, who appeared to be in a relative calm state during the experiment based on their facial expressions in the recorded videos. In addition, we assume that all participants have the design skills needed in the case, and that skills and emotions are not going to experience noticeable changes during a short period of time as skills gaining usually requires a relative long training process (Back et al., 2007; Gresham et al., 2001; Kazdin et al., 1992). Therefore, skills are treated as a stable parameter in our analysis. To sum up, participants have the same initial workload and their skills stay stable during the experiment.

It can be seen from Figure 1 that several possible states or factors are explored regarding the current object when breadth-first strategy is applied whereas only the current object is studied with depth-first strategy. Reif (Reif, 1985) argues that depth-first strategy is inherently sequential which means that the nodes from other branches are temporarily "forgotten". More branches of information can lead to more workload due to the different natures of the branches. From this point of view, designers seem to have less workload under depth-first process than breadth-first process by "forgetting" about other branches. However, as designers go deeper with a certain branch under depth-first strategy the target information becomes more and more specific which is difficult both to find and to understand. An increase in workload may be expected as designers continue with depth-first strategy.

In the meantime, within a depth-first process detailed information about the studied object will be collected which belongs to a certain domain. Depth-first process is more domain-specific than breadthfirst process and designer's domain knowledge is more likely to be activated during such a process. On the contrary, within a breadth-first process the successively studied objects are usually from the same level which tend to be less related to each other as with depth-first process. As those objects may belong to different fields and a wide range of knowledge is needed, designers are more likely to feel the shortage of knowledge with breadth-first process. It seems that more domain dependent knowledge will be activated with depth-first process than that with breadth-first process.

We may infer how designer's mental stress changes under breadth-first process and under depth-first process by integrating the aforementioned analysis into Eq. (1). With reduced workload and more activated domain knowledge, designer's mental stress may be lower when depth-first instead of breadth-first strategy is applied. This seems in accord with our sentiment that the depth-first approach can enhance the designer's mastering of the global picture of the design and thus reduce the feeling of uncertainty, which is closely related to stress. Furthermore, as participants were not given any instructions about information collection strategy we assume that designers would naturally adopt hybrid strategy. Design is a recursive process (Nguyen and Zeng, 2012; Zeng and Cheng, 1991) in which solutions to a sub-design problem may depend on those for another sub-problem. Any new pieces of design information (either of design problem, design solutions, and design knowledge) would reframe the problem, which could generate a new tree for 
search. As a result, though some designers may prefer either depth-first or breadth-first strategy, they would have to turn to the other strategy either because the current design needs information from another sub-design problem or because they cannot move deeper or wider with the available information. From this point of view, any application examples can be relatively seen as hybrid processes if the boundaries are defined as far as possible from the studied node. However, such hybrid processes may still have a dominant strategy, depth-first or breadth-first, as people may have a preference on one of them. And our previous analysis on these two information collection strategies may also apply for hybrid cases dominated by those two strategies. Therefore, our research hypothesis can be formulated as follows:

\section{- Hypothesis: Designer's mental stress in breadth-first dominant process is higher than that in depth-first dominant process.}

\section{CASE STUDY: “FLYING HOUSE” DESIGN EXPERIMENT}

\subsection{Description of the case}

The selected case comes from an experiment conducted by the Design Lab at Concordia University, which was previously used in a research work published in 2013 to study the distribution of mental stresses during conceptual design activities (Nguyen et al., 2013). In the experiment, six graduate students (four males and two females) with engineering background whose ages ranged from 25 to 35 years old were selected as participants. The experiment protocol was approved by Human Research Ethics and Compliance, Concordia University. HRV monitor-Polar RS800G3, tablet screen recorder and cameras (Figure 2) were used to record subject's physiological responses and design behaviours.

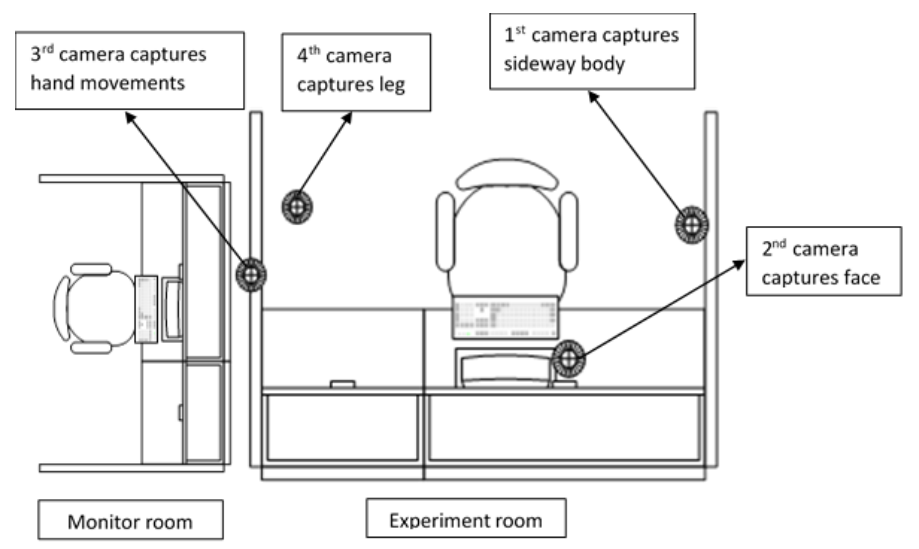

Figure 2. Camera settings

Each subject was assigned the same design task "design a house that can fly easily from one place to another place". They were given unlimited time to finish their design followed by a short interview. As mentioned in Section 2 that participants naturally apply certain type of information collection strategy without any instructions. The entire procedure of the experiments is as follows (Nguyen et al., 2013):

1. Subjects were asked to relax for 3 minutes with eyes open.

2. Subjects were asked to relax for another 3 minutes with eyes closed.

3. Subjects were given the predefined design problem and asked to solve it in unlimited time.

4. Subjects conducted the design using tablets with availability of the internet.

5. Subjects were asked to relax for 3 minutes with eyes closed after finishing their designs.

6. Subjects were interviewed.

\subsection{Case analysis and results}

The collected data were firstly segmented by applying protocol analysis. Besides observable actions shown in the screen recording video, subjects' answers during the interview stage were also used to calibrate the segmentation result. Moreover, several criteria were developed for recognizing the applied dominant strategy based on our analysis mentioned above and the analysis in (Wang et al., 2015) of three different information collection strategies. When breadth-first dominant strategy is applied, objects at the same level, which may belong to different branches, are studied. In the meantime, some closely related information may be collected repetitively while subjects investigate objects from the same branch 
at different stages of their design process. However, in a depth-first dominant process subjects may include more details in their solutions, which is enabled by the increase of certain domain knowledge. From another perspective, more efforts and time are required from the subjects whose solutions include more details than those with less detailed solutions. According to our discussions about relative hybrid process, we may use efforts and time as an indicator of one's preference to depth-first strategy. Therefore, the criteria used in our analysis to recognize the applied information collection strategy are as following: A sample is identified as a depth-first dominant process if it satisfies the following two conditions simultaneously: 1) there is little jumping between different branches; and 2) details are added for certain items. Otherwise, the sample is recognized as a breadth-first dominant process.

The collected HRV data were then analysed by HRVAS (Ramshur, 2010), a MATLAB software, with our customized adaptation. Discrete Wavelet Transform (DWT) was firstly applied to the data resulting in a series of DWT coefficients (Eq. (2)).

$$
C_{x}(m, n)=\frac{1}{2^{\frac{m}{2}}} \int_{-\infty}^{\infty} x(t) \Psi^{*}\left(\frac{t}{2^{m}}-n\right) d t
$$

where $\Psi^{*}(t)$ is the complex conjugate of the mother wavelet $\Psi(t), \alpha$ and $\tau$ are the scale and translation parameters, respectively. The WT coefficients were then used to compute the instantaneous band power. More specifically, the instantaneous power of a certain frequency interval was computed by integrating the squared modulus of the coefficients over $\left[f_{1}, f_{2}\right]$.

LF/HF ratio was computed from the computed band power of LF and HF. In terms of each extracted segment, the LF/HF ratio for a given segment was computed using the following equation (Eq. (3)):

$$
R_{j}=\frac{\sum_{i=1}^{n}\left(t_{i} \times r_{i}\right)}{\sum_{i=1}^{n} t_{i}}
$$

Thereafter, K-means was applied to the normalized LF/HF ratios in order to cluster the segment $\mathrm{LF} / \mathrm{HF}$ ratios of all subjects through minimizing the sum of absolute differences. As a result, all of the segments were classified into $\mathrm{K}$ different clusters corresponding to $\mathrm{K}$ different stress levels, with $\mathrm{K}$ varying from 2 to 12. This step helps to avoid the impact of duration time between and within samples, which means that two segments having the same LF/HF ratio will make the same contribution to the final results no matter how they are different in length. In addition, the percentage of segments identified at each stress level was computed for each subject, which could be understood as the stress distribution of each sample. Finally, a weighted average stress value was computed for each subject based on the obtained stress distribution. In other words, individual's stress was quantified with a one dimensional value which was calculated through multiplying the computed percentages by the centroids of their corresponding clusters (levels).

For each subject, his/her design process was divided into segments based on the result of protocol analysis. Figure 3 represents an example of the extracted segments.

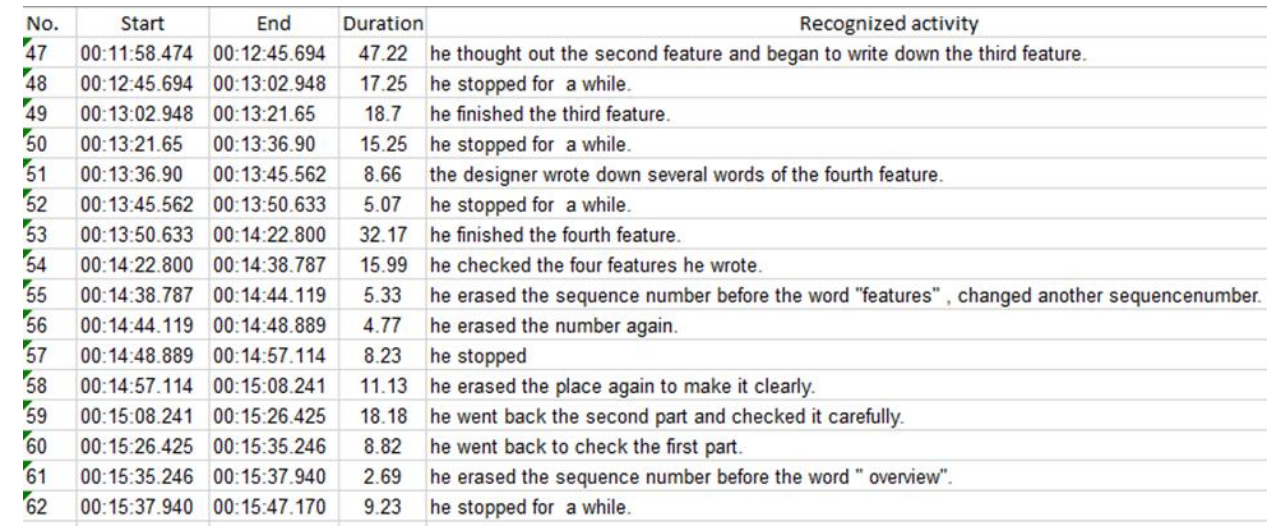

Figure 3. Part of the segmentation result of subject 4

According to the developed criteria for recognizing the dominant information collection strategy, three subjects were identified as depth-first dominant procedures and the other three as breadth-first dominant procedures (Table 1). More specifically, subject 1, 2 and 4 were recognized as depth-first (denoted as DF 
in Table 1) dominant while subject 3, 5 and 6 as breadth-first (denoted as BF in Table 1) dominant. The identified depth-first dominant processes tend to have smaller number of segments than breadth-first dominant processes. As one of the applied criteria for depth-first is "little jumping between branches", it seems reasonable that less jumping may lead to less segments within a depth-first process.

Based on the calculated WT coefficients, band power of LF and HF as well as the LF/HF ratios were computed for each subject (Figure 4). In addition, the detected peaks have been used to calibrate certain time intervals within segmentation results. For simplification, the mean value was computed as the (component) LF/HF ratio for each 10 -second window with $50 \%$ overlap before applying Eq. (4). More specifically, the component duration $t_{i}=5 \mathrm{~s}$ and $\mathrm{n}$ represents the number of 5 -second components in the studied segment.

Table 1. Segments and dominant strategy recognition results

\begin{tabular}{ccccccc}
\hline Subject ID & 1 & 2 & 3 & 4 & 5 & 6 \\
\hline Number of segments & 142 & 54 & 211 & 139 & 235 & 216 \\
\hline Dominant strategy & DF & DF & BF & DF & BF & BF \\
\hline
\end{tabular}

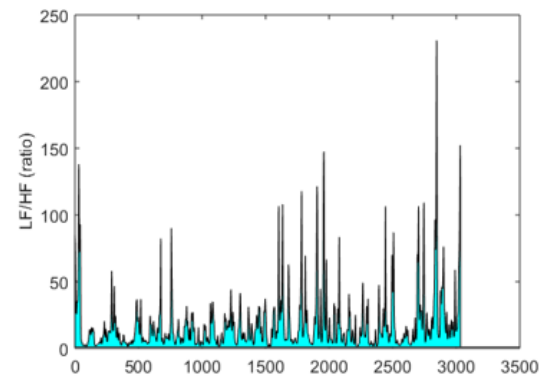

(a) Subject 1

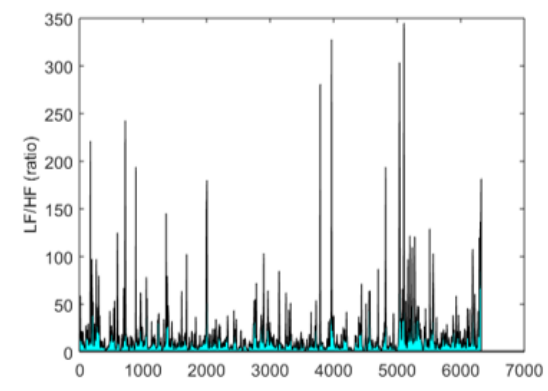

(c) Subject 3

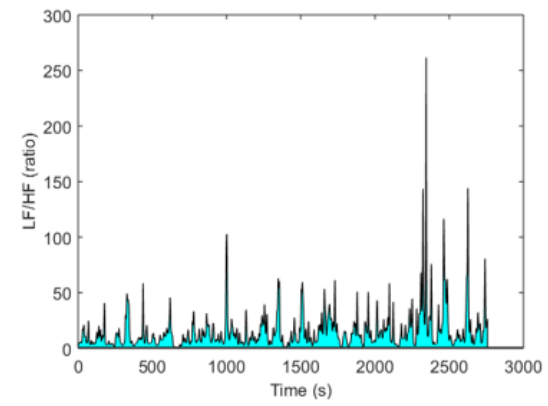

(e) Subject 5

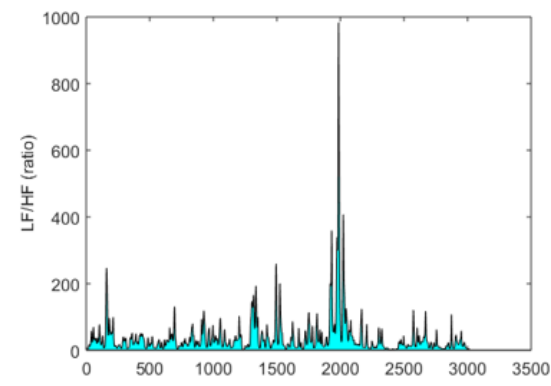

(b) Subject 2

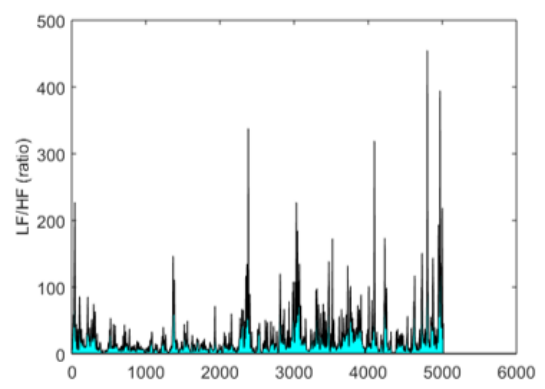

(d) Subject 4

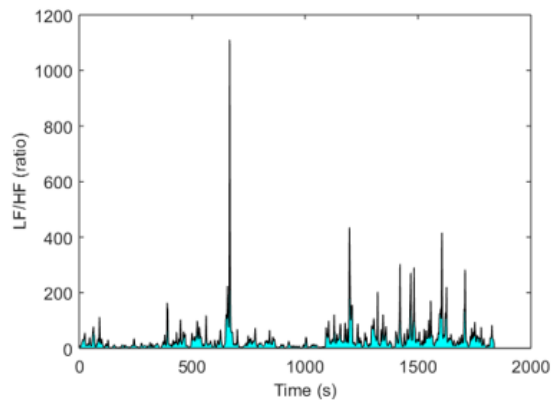

(f) Subject 6

Figure 4. Plotted LF/HF ratios for all subjects

Figure 5 depicts the distributions of the component LF/HF ratios for each subject. There is no significant difference between the distributions of each subject's mental stresses, except for subject 2. From the recorded video we noticed that Subject 2 has paid considerable attention to the detailed information related to aircraft, Airbus A380 in particular. This may be explained as a case of design fixation when designers are so attached to a certain field (Jansson and Smith, 1991; Linsey et al., 2010; Nguyen and Zeng, 2017). Another way to interpret this phenomenon is that the design process of this subject can be seen as a hybrid process dominated by depth-first strategy which so close to a pure depth-first process. In general, the plotted result seems to confirm our assumption that subjects naturally conduct a hybrid process where a 
similarity is expected in a considerable portion of the data whatever a dominant strategy is applied. Further analysis has been done to investigate the difference under breadth-first dominant and depth-first dominant hybrid processes during design activities.

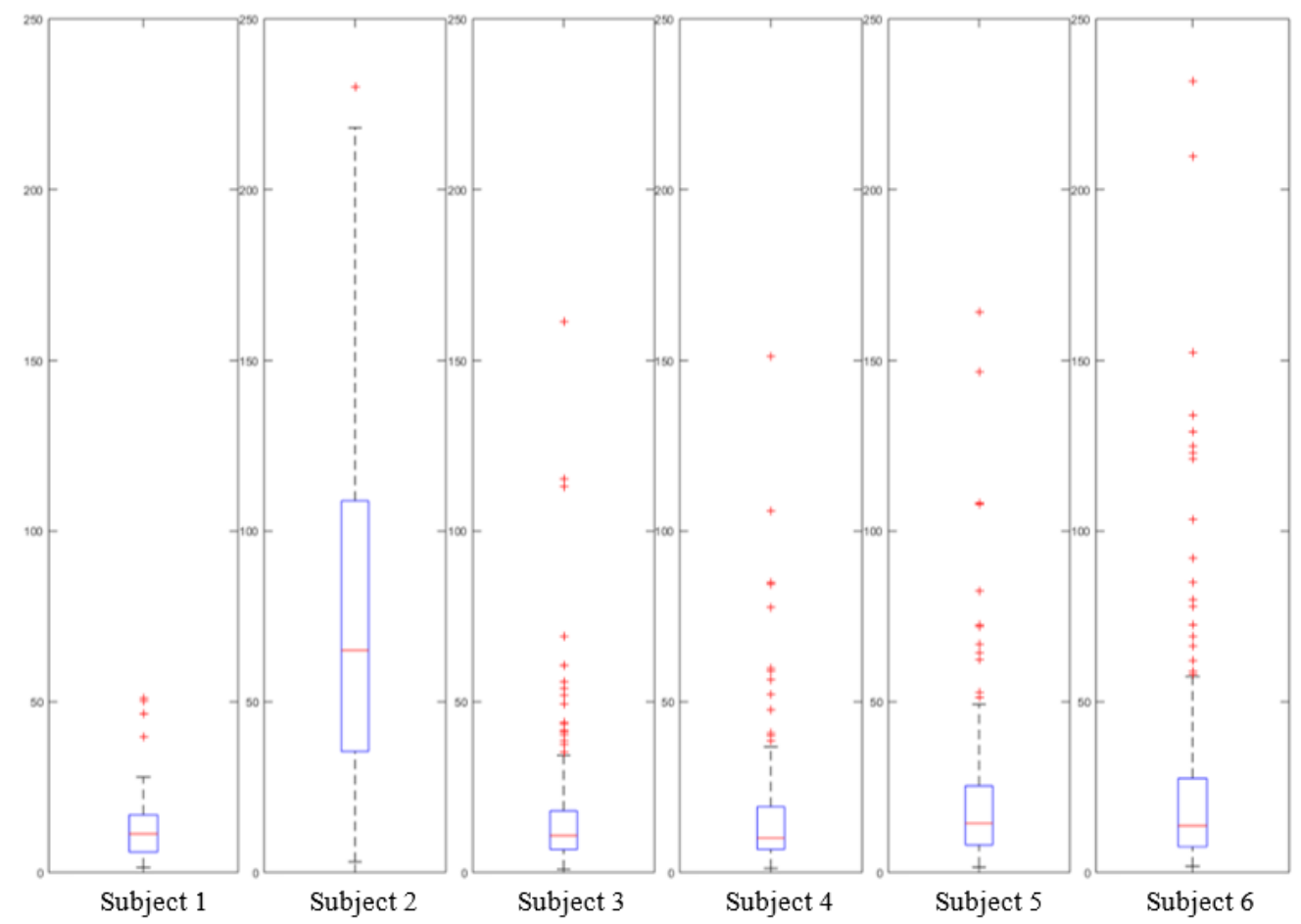

Figure 5. Distribution of $L F / H F$ ratios for all subjects
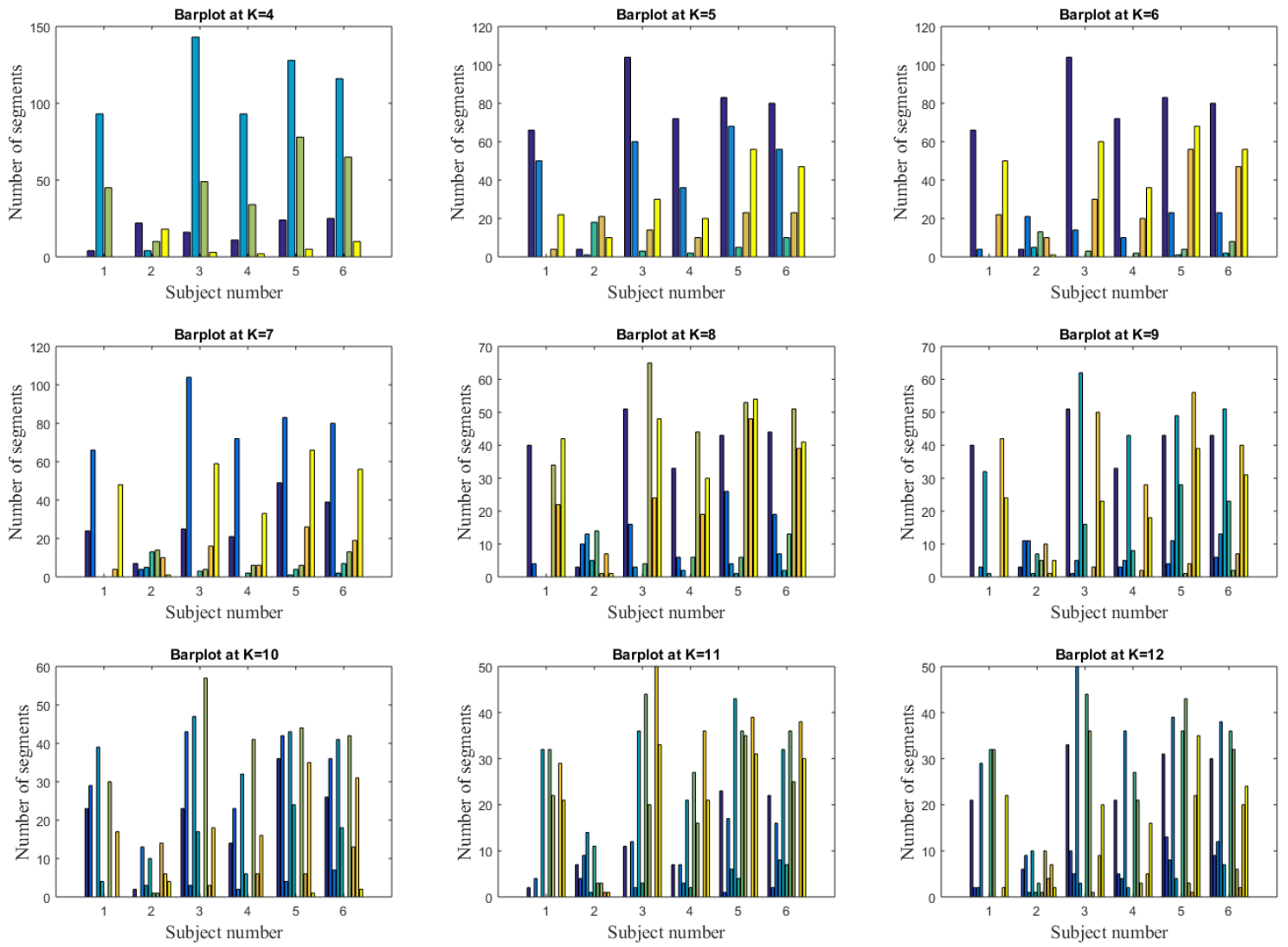

Figure 6. Segment distribution of each subject after $K$-means clustering $(K=4,5, \ldots, 12$; under a certain $K$ value, the $K$ different clusters are represented in $K$ different colours) 
After applying K-means to the normalized LF/HF ratios with $\mathrm{K}$ varying from 2 to 12 , the number of segments at different stress levels was computed for each subject. In the meanwhile, Friedman test was used to determine whether the difference between different clusters was significant given the small sample size. The p-values of Friedman test indicate that the difference between stress levels is not significant $(p>0.05)$ when segments are clustered into two $(p=0.1025)$ or three groups $(p=$ 0.0695). However, significant difference $(p<0.05)$ can be found between $\mathrm{K}$ different stress levels for $K \geq 4(K \leq 12)$. Therefore, we eliminated $K=2$ and $K=3$ when plotting subject's segment distribution (Figure 6) and stress values over $K$ (Figure 7).

A noticeable similarity can be found in the plotted segment distribution (Figure 6) between different subjects except for Subject 2, which is in accord with our findings from Figure 5. Different colours represent different clusters under a chosen $K$ value and the same colour is used to represent the same cluster for all subjects. As we can see from Figure 6, although $K$ changes from 4 to 12 the distribution of segments on each subject's data seem to follow a certain pattern for Subject 1, 3, 4, 5, and 6. According to the aforementioned analysis, design fixation happens with Subject 2 or depth-first strategy is applied. Despite Subject 2, participants' activities seem to have a certain similarity which is consistent with our assumption that designers naturally apply hybrid strategy for information collection.

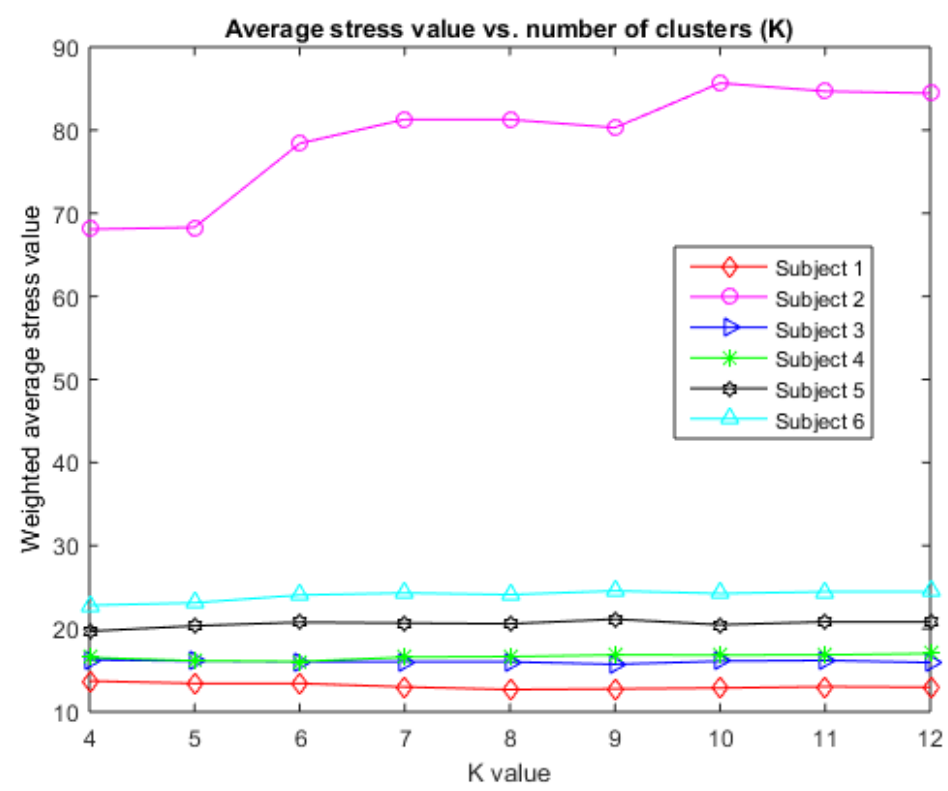

Figure 7. Weighted average stress values over varying $K$ values

Figure 7 shows each subject's weighted average mental stress at varying $K$ values. According to the results, subject's stress value varies as the number of cluster $(K)$ changes from 4 to 12 . Despite this fluctuation, the weighted average stress for each subject tends to remain stable at a certain stress value. As it can be noticed, the mental stress of one subject, Subject 2, is significantly higher than that of other subjects. As mentioned in the discussions of Figure 5, there are two ways to understand this phenomenon: 1) This subject was so attached to information related to aircraft, which can be seen as design fixation; and 2) According to the aforementioned assumption, the design process of this subject may be seen as a depth-first dominant hybrid process which is so close to a pure depth-first process where the "fly" branch is so deeply studied. For either reason, we should exclude Subject 2 for the following analysis as our hypothesis concentrates on hybrid process (without design fixation). Moreover, the mental stress values for the rest of subjects range from 12 to 25, among which the largest belongs to Subject 6 and the smallest belongs to Subject 1. By referring to the results of dominant strategy identification (Table 1), Subject 6 is identified as breadth-first dominant process which has higher mental stress than Subject 1 that is identified as depth-first dominant. This result seems in consistent with our developed hypothesis. However, the same conclusion cannot be achieved if we take all the studied samples into account. The computed average mental stresses for Subject 3 and Subject 4 are so close to each other and the former, which is identified as breadth-first dominant, seems to have lower stress for $K \geq 7$. Considering the limited sample size of this case study, further analysis needs to be done with increased samples to achieve a general conclusion. 


\section{CONCLUSION AND FUTURE WORK}

This work conducts a quantitative investigation of the relationship between information collection strategies and designer's mental stresses. This preliminary work uses data from six subjects participated in the experiment where videos and HRV data were recorded. The design process of each subject was segmented and the dominant information strategy was identified based on the observed behaviours from the videos. In the meanwhile, the collected HRV data were computed and clustered for the quantification of subject's mental stress during design processes. The case study results indicate that higher mental stress is more likely to happen to designers under a breadth-first dominant process than a depth-first dominant one. Moreover, it may be inferred that the breadth-first information collection strategy may lead to increased mental stress than that of the depth-first strategy during a design process, which may serve as the topic of follow-up studies.

Further studies are needed to provide more evidence to support the findings presented in this paper with larger sample size as there are only six subjects involved in the present case study. We will continue our research on this topic by comparing designers' mental stresses in pure depth-first and breadth-first processes, where they are instructed to follow a certain information collection strategy instead of making their natural choice. In addition, other physiological signals, such as EEG (electroencephalogram) and SC (skin conductance), apart from HRV, can be applied in future studies which may improve the quantification of mental stress and enable the quantification of designer's mental capacity.

\section{REFERENCES}

Back, A.L., Arnold, R.M., Baile, W.F., Fryer-Edwards, K.A., Alexander, S.C., Barley, G.E., Gooley, T.A. and Tulsky, J.A. (2007), "Efficacy of Communication Skills Training for Giving Bad News and Discussing Transitions to Palliative Care", Arch Intern Med, Vol. 167, pp. 453-460. https://doi.org/10.1001/archinte.167.5.453.

Bardwell, L.V. (1991), "Problem-Framing: A perspective on environmental problem-solving", Environmental Management, Vol. 15, pp. 603-612. https://doi.org/10.1007/BF02589620.

Belkin, N.J., Cool, C., Stein, A. and Thiel, U. (1995), "Cases, scripts, and information-seeking strategies: On the design of interactive information retrieval systems", Expert Systems with Applications, Vol. 9, pp. 379395. https://doi.org/10.1016/0957-4174(95)00011-W.

Boonnithi, S. and Phongsuphap, S. (2011), "Comparison of heart rate variability measures for mental stress detection, in: 2011 Computing in Cardiology", Presented at the 2011 Computing in Cardiology, pp. 85-88.

Broniarczyk, S.M. and Griffin, J.G. (2014), "Decision Difficulty in the Age of Consumer Empowerment", Journal of Consumer Psychology, Vol. 24, pp. 608-625. https://doi.org/10.1016/j.jcps.2014.05.003.

Cook, G.J. (1993), “An Empirical Investigation of Information Search Strategies with Implications for Decision Support System Design”, Decision Sciences, Vol. 24, pp. 683-698. https://doi.org/10.1111/j.15405915.1993.tb01298.x.

Eysenbach, G. and Köhler, C. (2002), "How do consumers search for and appraise health information on the world wide web? Qualitative study using focus groups, usability tests, and in-depth interviews", BMJ, Vol. 324, pp. 573-577. https://doi.org/10.1136/bmj.324.7337.573.

Gresham, F.M., Sugai, G. and Horner, R.H. (2001), "Interpreting Outcomes of Social Skills Training for Students with High-Incidence Disabilities”, Exceptional Children, Vol. 67, pp. 331-344. https://doi.org/10.1177/001440290106700303.

Healey, J.A. and Picard, R.W. (2005), "Detecting stress during real-world driving tasks using physiological sensors", IEEE Transactions on Intelligent Transportation Systems, Vol. 6, pp. 156-166. https://doi.org/10.1109/TITS.2005.848368.

Hjortskov, N., Rissén, D., Blangsted, A.K., Fallentin, N., Lundberg, U. and Søgaard, K. (2004), “The effect of mental stress on heart rate variability and blood pressure during computer work", Eur J Appl Physiol, Vol. 92, pp. 84-89. https://doi.org/10.1007/s00421-004-1055-z.

Jansson, D.G. and Smith, S.M. (1991), "Design fixation”, Design Studies, Vol. 12, pp. 3-11. https://doi.org/10.1016/0142-694X(91)90003-F.

Karthikeyan, P., Murugappan, M. and Yaacob, S. (2011), "ECG signals based mental stress assessment using wavelet transform", in: 2011 IEEE International Conference on Control System, Computing and Engineering. Presented at the 2011 IEEE International Conference on Control System, Computing and Engineering, pp. 258-262. https://doi.org/10.1109/ICCSCE.2011.6190533

Kazdin, A.E., Siegel, T.C. and Bass, D. (1992), "Cognitive problem-solving skills training and parent management training in the treatment of antisocial behavior in children", Journal of Consulting and Clinical Psychology, Vol. 60, pp. 733-747. https://doi.org/10.1037/0022-006X.60.5.733. 
Kumar, M., Weippert, M., Vilbrandt, R., Kreuzfeld, S. and Stoll, R. (2007), "Fuzzy Evaluation of Heart Rate Signals for Mental Stress Assessment”, IEEE Transactions on Fuzzy Systems, Vol. 15, pp. 791-808. https://doi.org/10.1109/TFUZZ.2006.889825.

Leber, A.B. and Egeth, H.E. (2006), "It's under control: Top-down search strategies can override attentional capture”, Psychonomic Bulletin \& Review, Vol. 13, pp. 132-138. https://doi.org/10.3758/BF03193824.

Linsey, J.S., Tseng, I., Fu, K., Cagan, J., Wood, K.L. and Schunn, C. (2010), “A study of design fixation, its mitigation and perception in engineering design faculty”, J. Mech. Des, Vol. 132, 041003-041003-12. https://doi.org/10.1115/1.4001110

Nguyen, T.A., Xu, X., Zeng, Y. (2013), "Distribution of mental stresses during conceptual design activities", DS 75-7: Proceedings of the 19th International Conference on Engineering Design (ICED13), Design for Harmonies, Vol. 7: Human Behaviour in Design, Seoul, Korea, 19-22.08.2013.

Nguyen, T.A. and Zeng, Y. (2017), “A theoretical model of design fixation”, International Journal of Design Creativity and Innovation, Vol. 5, pp. 185-204. https://doi.org/10.1080/21650349.2016.1207566.

Nguyen, T.A. and Zeng, Y. (2012), “A theoretical model of design creativity: Nonlinear design dynamics and mental stress-creativity relation”, Journal of Integrated Design and Process Science, Vol. 16, pp. 65-88.

Ramshur, J.T. (2010), Design, evaluation, and application of Heart Rate Variability Analysis Software (HRVAS), University of Memphis.

Reif, J.H. (1985), "Depth-first search is inherently sequential”, Information Processing Letters, Vol. 20, pp. 229234. https://doi.org/10.1016/0020-0190(85)90024-9.

Russo, J.E. and Dosher, B.A. (1983), "Strategies for multiattribute binary choice”, J Exp Psychol Learn Mem Cogn, Vol. 9, pp. 676-696.

Salahuddin, L., Cho, J., Jeong, M.G., Kim, D. (2007), "Ultra Short Term Analysis of Heart Rate Variability for Monitoring Mental Stress in Mobile Settings", in: 2007 29th Annual International Conference of the IEEE Engineering in Medicine and Biology Society. Presented at the 2007 29th Annual International Conference of the IEEE Engineering in Medicine and Biology Society, pp. 4656-4659. https://doi.org/10.1109/IEMBS.2007.4353378

Scott, T.J. and O’Sullivan, M.K. (2005), “Analyzing Student Search Strategies: Making a Case for Integrating Information Literacy Skills into the Curriculum”, Teacher Librarian, Vol. 33 No. 21.

Vu, T.M.H., Tu, V.P. and Duerrschmid, K. (2016), "Design factors influence consumers' gazing behaviour and decision time in an eye-tracking test: A study on food images", Food Quality and Preference, Vol. 47, pp. 130-138. https://doi.org/10.1016/j.foodqual.2015.05.008.

Wang, X., Nguyen, T.A., Zeng, Y. (2015), "Influence of information collection strategy in problem formulation on design creativity through mental stress: a theoretical analysis", in: DS 80-11 Proceedings of the 20th International Conference on Engineering Design (ICED 15) Vol 11: Human Behaviour in Design, Design Education; Milan, Italy, 27-30.07. 15. pp. 91-100.

Wilczynski, N.L. and Haynes, R.B. (2004), "Developing optimal search strategies for detecting clinically sound prognostic studies in MEDLINE: an analytic survey", BMC Medicine, Vol. 2 No. 23, https://doi.org/10.1186/1741-7015-2-23.

Zeng, Y. (2004), “Environment-based formulation of design problem”, J. Integr. Des. Process Sci, Vol. 8, pp. 45-63.

Zeng, Y. and Cheng, G.D. (1991), “On the logic of design”, Design Studies, Vol. 12, pp. 137-141. https://doi.org/10.1016/0142-694X(91)90022-O.

\section{ACKNOWLEDGEMENTS}

This reported research is supported by the NSERC Discovery Grant, NSERC Charis in Design Engineering Program and NSERC CRD project. The authors thank Xiaoying Wang for her early involvement and all the volunteers for their participation during the experiment. 